\title{
Enhanced Fault Detection and Isolation in Modern Flight Actuators
}

\author{
Daniel Ossmann*
}

\begin{abstract}
Due to their central location in the control system, actuation systems of primary control surfaces in modern, augmented aircraft must show an increased reliability. A traditional approach is based on hardware redundancy. In this way, modern actuation systems of one single control surface consist of up to two actuators and three sensors. These different dynamic subsystems are all prone to faults themselves and can be monitored. This paper presents the setup of a fault detection and diagnosis (FDD) system to systematically detect and isolate faults in the subcomponents of an actuation system. Based on the achievable fault signature matrix of the system, a residual filter is designed using nullspace theory. The residuals of the proposed filter form the basis of the decision making process to detect an isolate the faults. The developed FDD system is implemented into a nonlinear aircraft model, allowing a profound validation of the FDD system's detection and isolation performance for different actuator and sensor fault scenarios.
\end{abstract}

\section{INTRODUCTION}

To comply with the safety regulations, the actuation system of a single control surface in modern aircraft includes not only one actuator and one sensor. Up to three sensors and two actuators are integrated to be able to guarantee a backup during fault situations. In detail, the redundant actuator is usually in a passive mode and is activated only when any fault in the system is detected [1], while the faulty actuator is switched off. If this switching does not improve the situation, the whole actuation system of the control surface is switched off. The detection of actuator faults is a widely discussed topic in literature. For example in [1] a nonlinear observer based approach is used to detect oscillatory failures of the actuator on system level (local), while [2] proposes a combination of a local and aircraft system wide (global) approach to detect actuator faults. Pure global approaches are provided e.g. by [3] and [4]. While various approaches are able to detect faults in an actuation system quite well, none of them tries to distinguish between physical faults of the actuator and faulty sensors, necessary in the actuation system to close the actuator's control loop. This lack of sufficient isolation information leads to the common procedure in modern, augmented aircraft: as soon as any fault is detected on the actuator, this actuator is switched off and the redundant actuator is activated, no matter if the actuator itself or a single sensor causes the faulty behavior. This paper proposes an approach, which is not only able to detect but also to isolate faults on local actuator level. In case of a sensor fault the isolation information can be used

\footnotetext{
* Resarch Associate, Department of Aircraft systems dynamics, Institute of System Dynamics and Control, DLR Oberpfaffenhofen, D-82234 Wessling, Germany; daniel.ossmann@dlr.de
}

to switch to a redundant physical or virtual sensor, without losing the physical actuator redundancy.

In section II a common model of an electro-hydraulic aircraft actuator is presented, including the modeling of the actuator and sensor faults. A linear model of the actuator description is derived enabling the design of the residual filter. In section III, based on the achievable fault signature matrix (AFSM) of the system, the most robust structure for the desired fault signature matrix (DFSM) is selected for the synthesis process of the residual filter. To robustly solve the fault detection and isolation problem (FDIP) a linear parameter varying (LPV) form of the designed filter is determined. In section IV the residual evaluation and decision making algorithms are discussed to complete the setup of the FDD system. This finally enables in section VI the validation of the FDD system with a nonlinear aircraft model, including a nonlinear model of the actuation system.

\section{THE FDD SYSTEM FOR FAULT DETECTION AND ISOLATION}

The FDD system to be designed able to detect and isolate actuator and sensor faults is depicted in Fig. 1: The FDD system consists of the residual generator, the residual evaluator and the decision making function. The residual

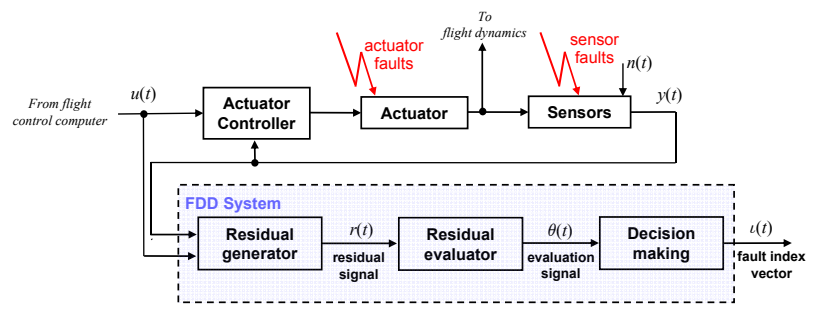

Fig. 1. FDD system for detection and isolation of faults in an actuation system

generator for fault detection and isolation in Fig. 1 processes the commanded position $u$ and the measurement signals $y$ to generate the residual signal vector $r$. The residual evaluator computes a specific approximation $\theta_{j}$ of each individual residual norm $\left\|r_{j}\right\|$. These values are used in the decision making process, where a threshold-based decision logic is employed to generate the fault signature vector $\varsigma$. This vector is compared to the columns of the fault signature matrix $S$, in which a unique signature for each fault is coded, finally enabling the determination the fault index vector $\iota$ and isolate the occurring fault situation. The employed based 
methodology for the design of the elements of the FDD system in Fig. 1 has the following main steps:

1) Development of suitable synthesis models of the underlying actuator

2) Synthesis of the residual generator for robust fault detection based on the achievable fault signature matrix $M$

3) Setting up of the residual evaluation and decision making functions

In what follows these steps are described in more detail.

\section{ACTUATION SYSTEM MODEL}

The actuation system consists of a hydraulic servo controlled actuator, an actuator in passive mode, and three sensors, one on each rod of the two actuators and one on the control surface. This actuation system can be analytically described by a higher order model presented in [5]. The underlying model of the actuation system used in this paper is a simplified model, which is commonly used for the simulation and analysis of aircraft actuators [1]. It is described by the first order nonlinear state equation

$$
\dot{x}=k_{c i} i_{c} \sqrt{\frac{\Delta P(x)-\frac{F_{a e r o}(p, x, \dot{x})+k_{d} \dot{x}^{2}}{s}}{\Delta P_{\text {ref }}}},
$$

with the control law $i_{c}=k_{p}(u-x)$. In this equation $k_{p}$ is the servo control gain, $k_{c i}$ is a gain to convert an estimated current to a corresponding rod speed, $\Delta P$ is the hydraulic pressure delivered to the actuator, $\Delta P_{r e f}$ is a differential pressure for a fully opened servo-valve (maximum rod speed), $F_{\text {aero }}$ represents the aerodynamic force at the control surface, $k_{d} \dot{x}^{2}$ an estimate of the servo-control load induced by the adjacent actuator in damping mode and $s$ is the actuator piston surface area. The aerodynamic force $F_{\text {aero }}$ depends on the position and the velocity of the actuator and additionally on measurable and nonmeasurable flight parameters summarized in the vector $p$. This dependency usually cannot be analytically described for the whole flight envelope and is given in a higher-dimensional table only. Hence, the model (1) cannot be implemented on flight computers. However, in equation (1) the nonlinear gain

$$
k_{a}(p, x, \dot{x}):=k_{c i} \sqrt{\frac{\Delta P(x)-\frac{F_{a e r o}(p, x, \dot{x})+k_{d} \dot{x}^{2}}{s}}{\Delta P_{\text {ref }}}}
$$

can explicitly be determined and used together with the control law in the first order quasi-LPV state equation

$$
\dot{x}=-k_{p} k_{a}(\rho) x+k_{p} k_{a}(\rho) u .
$$

The term $k(\rho)=k_{p} k_{a}(\rho)$, with $\rho=(x, \operatorname{sign}(\dot{x}), p)$, has been approximated in [6] by an analytical function, only depending on measurable parameters. Together with (3) this approximation of the actuator dynamics can be used in model-based fault diagnosis applications [6] and implemented on flight computers. The output function $y$ of the model (3) includes three measurement signals. Due the physical connection of the two actuators, it is assumed that the rod potions of both actuators are equal. However, their measurement signals $y_{1}$ and $y_{3}$ are influenced by different stochastic noise influences. The deflection of the control surface has a proportional relation $k_{f}$ with the rod position $x$. The resulting output function is given by

$$
y=\left[\begin{array}{lll}
1 & k_{f} & 1
\end{array}\right]^{T} x+n,
$$

where $n$ is a stochastic noise vector.

\section{A. Linear approximation with faults}

To enable a detailed analysis of the underlying system with faults, the actuator model is enhanced by $m_{f}=4$ fault situations: first, the nominal control law $i_{c}=k_{p}(u-x)$ is enhanced to

$$
i_{c}=k_{p}(u-x)+f_{a},
$$

where $f_{a}$ is an additive fault used to describe actuator faults. For example, with $f_{a}=A_{f} \sin \left(\omega_{f} t\right)$, where $A_{f}$ is the fault amplitude and $\omega_{f}$ the fault frequency, an oscillatory failure case can be modeled. The enhanced output function of the actuator model, including three sensor faults, is given by

$$
y=\left[\begin{array}{lll}
1 & k_{f} & 1
\end{array}\right]^{T} x+n+f_{s} .
$$

The additive sensor fault vector $f_{s}$ allows the modeling of three sensor faults $f_{s, 1}, f_{s, 2}$ and $f_{s, 3}$. For example a freezing of the third sensor at the position $c_{f}$ is created by selecting $f_{s, 3}=-x+c_{f}$.

Setting the parameters $\rho$ in (3) to constant values and including the defined fault and noise inputs leads to the first order linear model of the actuation system

$$
\begin{aligned}
& \dot{x}=-k_{p} k_{a} x+k_{p} k_{a} u+k_{a} f_{a} \\
& y=\left[\begin{array}{lll}
1 & k_{f} & 1
\end{array}\right]^{T} x+n+f_{s} .
\end{aligned}
$$

Fig. 2 shows a simplified block diagram of this model, including the different fault and noise inputs. This description of the actuator will serve as basis for the residual generator design. At the end of the design procedure an approach for the enhancement to a robust residual filter will be given.

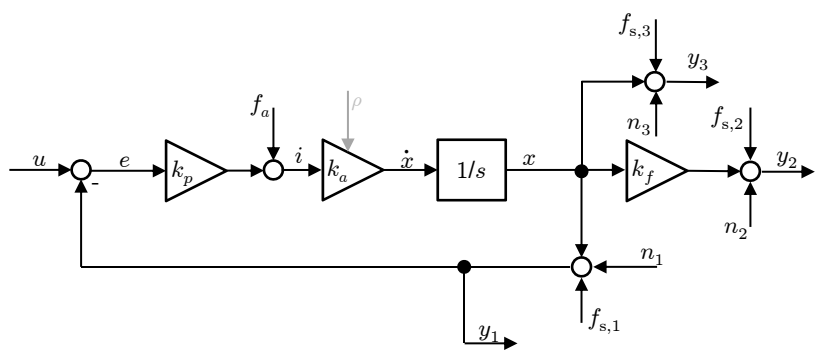

Fig. 2. Simplified block diagram of the actuator

\section{Synthesis of the Residual Generator}

The linear system (7) can also be written in the inputoutput form

$$
\mathbf{y}(s)=G_{u}(s) \mathbf{u}(s)+G_{f}(s) \mathbf{f}(s)+\mathbf{n}(s),
$$


where $\mathbf{y}(s), \mathbf{u}(s), \mathbf{f}(s)$ and $\mathbf{n}(s)$ are the Laplace-transformed quantities of $y(t), u(t), f(t)=\left[f_{a}(t) f_{s}(t)\right]^{T}$ and $n(t)$, respectively, and $G_{u}(s)$ and $G_{f}(s)$ are the corresponding transfer matrices. To solve the fault detection and isolation problem for the system (8) the residual filter

$$
\mathbf{r}(s)=Q(s)\left[\begin{array}{c}
\mathbf{y}_{1}(s) \\
\mathbf{y}_{2}(s) \\
\mathbf{y}_{3}(s) \\
\mathbf{u}(s)
\end{array}\right]
$$

shall be generated. $\mathbf{y}_{1}(s), \mathbf{y}_{2}(s), \mathbf{y}_{3}(s)$ are the entries of $\mathbf{y}(s)$ and $\mathbf{r}(s)$ is the Laplace-transformed quantity of the residual $r(t)$. For a physically realizable filter, $Q(s)$ must be proper and stable, having only poles with negative real parts.

To achieve the desired fault isolation, a bench of residual generators $Q^{j}(s)$ is designed, using the methods described in [6], where each of the filter shall generate a unique fault to residual influence vector. Each of these vectors describes the influence of the defined faults on one residual. The overall residual generation system consists of $q$ individual residual filters, which are designed separately and assembled to one system at the end according to

$$
Q(s)=\left[\begin{array}{c}
Q^{1}(s) \\
\vdots \\
Q^{q}(s)
\end{array}\right]
$$

To gain the knowledge how to design this set of filters requires the definition of the fault signature matrix (FSM) is required.

\section{A. Fault signature matrix}

The FSM $S$ of a residual filter describes the effects of the faults on the residuals. Let $R_{f_{i}}^{j}(s)$ be the transfer function of the $i$-th fault to the $j$-th residual. Then the entries of $S$ are defined as

$$
M_{j, i}= \begin{cases}1 & \text { if } R_{f_{i}}^{j}(0) \neq 0 \\ -1 & \text { if } R_{f_{i}}^{j}(0)=0 \text { and } R_{f_{i}}^{j}(s) \neq 0 \\ 0 & \text { if } R_{f_{i}}^{j}(s)=0 .\end{cases}
$$

In words, the entry ' 1 ' indicates a stationary influence of the fault to the residual (strong detection), the entry ' 0 ' indicates decoupled behavior of the residual from the fault, and the entry '-1' indicates that the residual is influenced by the fault, but its steady state value is 0 (weak detection).

The common way to design a residual generator is as follows: select a DFSM $\bar{S}$ and try to design the residual filter. The matrix $\bar{S}$ is achievable, if the design has been successful and the residual filter shows a FSM $S=\bar{S}$. In this paper we propose a more straight forward approach: determine the overall $\bar{q} \times m_{f}$ AFSM $M$ of a system before the synthesis of the residual filter. Here, $m_{f}$ is the number of defined faults and $\bar{q}$ defines the maximum number of different fault specifications vectors. This approach adds the design freedom of selecting the most robust structure for the DFSM $\bar{S}$ by selecting and arranging the desired rows of $M$. The filter is designed in a systematic way with a reduced number of design iterations. A systematic approach to determine the AFSM of a given system is presented in [7].

To answer the question how to adequately select the rows of $M$ for the residual filter synthesis, the robustness issues of the fault isolation process have to be discussed: The robustness of fault isolation concerns mainly so-called false firing and partial firing [8]. False firing is the case, when a residual exceeds its threshold in a situation, where it is supposed to stay below. This may happen due to unexpected noise/disturbances or inputs and faults acting on the residual due to uncertainties in the underlying system, when an exact decoupling is not possible any more. Partial firing appears, when a fault occurs and some residuals stay below their thresholds which they are supposed to exceed. This may simply happen due to a too high threshold. In both cases the correct fault cannot be isolated: The isolation requires an exact match of the generated so-called fault signature vector with the corresponding column of the signature matrix $S$. The fault signature vector is a $q$-dimensional vector, indicating which residual has exceeded its threshold (entry is set to 1) and which residual has not (entry is 0 ). Hence, it can happen, that a wrong fault signature vector for a fault matches another column in $S$ and thereby isolates the wrong fault. To avoid such wrong isolations [8] proposes to use unidirectional strong isolating structures. Such structures are characterized as follows: All columns of an unidirectional strong isolating structure are different and no column can be transferred into another by turning an arbitrary number of '1's into '0's or an arbitrary number of '0's into '1's. This requires for each pair of columns of a matrix a row where one column has a '1'-entry and the other column has a '0'-entry and vice versa.

The described procedure is now applied to the actuator model. First, for the linear system (7) the AFSM is determined:

$$
M=\left[\begin{array}{cccc}
1 & 1 & 1 & 1 \\
-1 & 0 & 0 & 1 \\
1 & 1 & 0 & 1 \\
1 & 0 & 1 & 1 \\
0 & 1 & 1 & 0 \\
-1 & 1 & 1 & 1 \\
0 & -1 & 0 & 1 \\
0 & 0 & -1 & 1 \\
0 & -1 & -1 & 1 \\
1 & 1 & 0 & 0 \\
1 & 0 & 1 & 0 \\
1 & 1 & 1 & 0
\end{array}\right] .
$$

The first three columns of the matrix correspond to the sensor faults while the last column corresponds to the actuator fault. This matrix directly indicates that the fault detection and strong isolation problem, where each residual is just influenced by one single fault and is decoupled from all other faults, is not solvable. This would require a $4 \times 4$ identity matrix, which cannot be generated using the rows of (12). However, the fault detection and weak isolation problem can be solved. Solving this problem leads to a residual filter which allows the isolation of all faults. The 
main disadvantage of weak isolation compared to strong isolation is, that it has to be assumed that faults occur one at a time [7].

For the residual filter design, a subset of the rows of $M$ is selected. The only way to generate a unidirectional strong isolating structure out of (12) is to include one row showing a -1-entry:

$$
\bar{S}=\left[\begin{array}{cccc}
1 & 1 & 0 & 1 \\
1 & 0 & 1 & 1 \\
1 & 1 & 0 & 0 \\
1 & 0 & 1 & 0 \\
1 & 1 & 1 & 0 \\
0 & 1 & 1 & 0 \\
0 & 0 & -1 & 1
\end{array}\right]
$$

-1-entries should usually be avoided for the design of the residual filter, as they may cause problems during the detection. As the stationary value of the step response is equal to zero, no clear correspondence to the columns of $S$ is given for a constant fault. However, a closer look at the first 6 rows of the matrix reveals that the only pair which is missing to make the matrix unidirectional strong isolating is a ' 0 '-entry in the first column combined with an '1'-entry in the fourth column, which is achieved by the last row in (13). Hence, the first six rows can be used to isolate the faults $f_{s, 2}$ and $f_{s, 3}$ by comparing the generated fault signature vector to the columns of $S$, while all seven rows of the vector must agree to either the first or fourth column of (13) to isolate the faults $f_{s, 1}$ or $f_{a}$ respectively. Using this approach the -1-entry does not cause any problems.

\section{B. Residual Generator}

As the $7 \times 4$ DFSM of the residual filter is known, it is possible to design the bank of residual generators $Q^{j}(s)$, with $j=1, \ldots, 7$. For the DFSM in (13), the bank of residual filters have to be designed that for all command inputs $u(t)$

(i) for $j=1, \ldots, 7, r_{j}(t)=0$ if $f_{i}(t)=0, \forall j$ with $\bar{S}_{j, i} \neq 0$

(ii) for $j=1, \ldots, 7, r_{j}(t) \neq 0$ if $f_{i}(t) \neq 0, \forall i$ with $\bar{S}_{j, i} \neq 0$

(iii) all residuals $r_{j}(t)$ are asymptotically bounded

with $i=1, \ldots, 4$ is fulfilled. In words, each detector $Q^{j}(s)$ has to achieve the desired signature of the $j$-th row of $\bar{S}$ and decouple the residual from the command input. One possible linear detector for the system (7) fulfilling the conditions (i) $-($ iii $)$ can be determined as

$$
\begin{aligned}
& Q(s)= \\
& {\left[\begin{array}{ccc}
0 & -\frac{a}{s+a} \frac{s+k_{p} k_{a}}{k_{f} k_{p} k_{a}} & 0 \\
0 & 0 & -\frac{a}{s+a} \frac{s+k_{p} k_{a}}{k_{p} k_{a}} \\
k_{f} \frac{a}{s+a} & -\frac{a}{s+a} & 0 \\
\frac{a}{s+a} & 0 & -\frac{a}{s+a} \\
-\frac{a}{s+a} & \frac{2}{k_{f}} \frac{a}{s+a} & -\frac{a}{s+a} \\
0 & \frac{a}{s+a} & -k_{f} \frac{a}{s+a} \\
k_{p} k_{a} \frac{a}{s+a} & 0 & \frac{a s}{s+a}
\end{array}\right.}
\end{aligned}
$$

where the parameter $a$ is an arbitrary number above zero, defining the transfer dynamic of the faults to the residuals. The detector has been determined analytically, where the main ideas are described below:

The first two rows as well as the last row of $Q(s)$ use a model of the actuator dynamics to determine the residuals, as their entries of the last column corresponding to the input signal $u$, are nonzero. For example, the first row of (14) multiplies the measurement signal $y_{2}$ with the inverted, fault and noise free actuator dynamics $\frac{s+k_{p} k_{a}}{k_{f} k_{p} k_{a}}$. The resulting signal is a kind of virtual input $\hat{u}$ which is subtracted from the real input $u$ to determine the first residual. Due to this subtraction the residual is decoupled from any input. The transfer function $\frac{a}{s+a}$ is used to make the transfer behavior from the faults to the residual proper. As the faults $f_{s, 1}, f_{s, 2}$ and $f_{a}$ directly influence the measurement signal $y_{2}$ and thus $\hat{u}$ but not $u$, the coupling of the faults with the residual is maintained. Hence, the transfer characteristics of the first row of (14) correspond to the one coded in the row of the DFSM in (13). The last two rows of the detector (14) are designed following the same ideas. The other four rows of $Q(s)$ mainly make use of signal based residual generation, as they only use the three measurement signals, which are subtracted from each other to achieve the decoupling from $u$. The proportional gain $k_{f}$ is needed to transfer the rod position to the surface deflection.

To enable a real-time implementation, it is often necessary to transfer the fault detection filter into the time domain. The state space matrices of the detector (14) are given by:

$$
A=-a I_{7}
$$

$$
B=\left[\begin{array}{cccc}
0 & \frac{a}{k_{f}}\left(1-\frac{a}{k_{p} k_{a}}\right) & 0 & a \\
0 & 0 & a\left(1-\frac{a}{k_{p} k_{a}}\right) & a \\
k_{f} a & -a & 0 & 0 \\
a & 0 & -a & 0 \\
-a & \frac{2 a}{k_{f}} & -a & 0 \\
0 & a & -k_{f} & 0 \\
k_{p} k_{a} a & 0 & -a^{2} & -k_{p} k_{a} a
\end{array}\right]
$$

$C=I_{7}$

$$
D=\left[\begin{array}{cccc}
0 & -\frac{a}{k_{f} k_{p} k_{a}} & 0 & 0 \\
0 & 0 & -\frac{a}{k_{p} k_{a}} & 0 \\
0 & 0 & 0 & 0 \\
0 & 0 & 0 & 0 \\
0 & 0 & 0 & 0 \\
0 & 0 & 0 & 0 \\
0 & 0 & a & 0
\end{array}\right]
$$

This form of the filter enables also the introduction of a more robust fault detection filter. In matrices $B$ resp. $D k_{a}$ can be replaced by a parameter-dependent description $k_{a}(\rho)$ of the actuator gain to robustly solve the fault detection and isolation problem in the whole flight envelope. Fig. 3 shows the step responses of the overall residual generation system (linear plant model together with the residual filter). In this example the parameter $a$ is selected at a value of $a=5$, while the parameters of the actuator are given at 
$k_{p}=2, k_{a}=7.5$ and $k_{f}=1.3$. The nominal case is shown in green clearly indicating that the behavior defined in the DFSM is achieved $(S=\bar{S})$. Additionally uncertainties in the parameter $k_{a}$ of the linear actuator model to be monitored of up to $25 \%$ are considered. The results are depicted in blue. However, these amplitudes are smaller than the amplitudes induced by the different faults, enabling the selection of an adequate threshold to separate the faults from undesired disturbances. Note, that only residuals based on a filter which uses a model of the actuator dynamics are influenced by these uncertainties. As the uncertainties directly influence all measurements, the decoupling from $u$ can be achieved in case of the signal based residual generation. In these cases the exact decoupling is not achieved any more, leading to small amplitudes of the residuals.

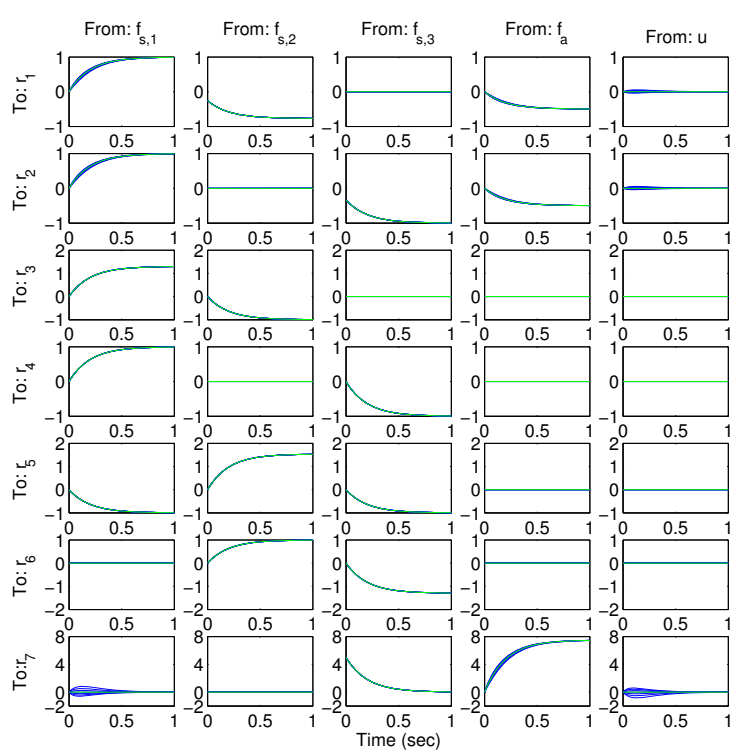

Fig. 3. Step responses of the actuator input and the possible faults to the residuals

\section{SETUP of RESIDUAL EVAluation AND DECISION} MAKING BLOCKS

\section{A. Residual evaluation}

The evaluation of residual signals often requires the computation of measures of the signals' energy, for which the 2-norm of a signal is usually an appropriate choice. For this purpose, a so-called Narendra signal evaluation scheme of the form

$$
\theta_{j}(t)=\alpha_{j}\left|r_{j}(t)\right|+\beta_{j} \int_{0}^{t} e^{-\gamma_{j}(t-\tau)}\left|r_{j}(\tau)\right| d \tau,
$$

can be used. The filter parameters $\alpha_{j} \geq 0$ and $\beta_{j} \geq 0$ are suitable weights for instantaneous and long-term values, respectively, while $\gamma_{j}>0$ is the forgetting factor.

\section{B. Decision making}

Each evaluation signal $\theta_{j}(t)$ is compared to a specific threshold $J_{t h, j}$ in the decision making process using the decision logic

$$
\begin{aligned}
& \theta_{j}(t)<J_{t h, j} \Rightarrow \varsigma_{j}(t)=0 \\
& \theta_{j}(t) \geq J_{t h, j} \Rightarrow \varsigma_{j}(t)=1
\end{aligned}
$$

to determine the components of the fault signature vector $\varsigma(t)$. Like the residual $r_{j}(t)$, the evaluated residual $\theta_{j}(t)$ is ideally equal to zero or sufficiently small in fault free situations or in fault situation where the corresponding residual is decoupled from the fault. It shall exceed the threshold $J_{t h, j}$ when a fault occurs, from which the residual is not decoupled. Hence, the appropriate selection of the values of the free parameters $\alpha_{j}, \beta_{j}$ and $\gamma_{j}$, together with an appropriate threshold $J_{t h, j}$ essentially influences the performance of the FDD system. Optimization based approaches how to determine the values of the parameters can be found in [6], [10].

As discussed in section IV-A, the fault situations are coded in the columns of the FSM $S$ :

$$
S_{\left(q \times m_{f}\right)}=\left[\begin{array}{ccc}
s_{1,1} & \cdots & s_{1, m_{f}} \\
\vdots & \ddots & \vdots \\
s_{q, 1} & \cdots & s_{q, m_{f}}
\end{array}\right]=\left[\begin{array}{lll}
\bar{v}_{1} & \ldots & \bar{v}_{m_{f}},
\end{array}\right]
$$

where every column $\bar{v}_{i}$ has an individual structure of zeros and ones. Hence, to isolate a fault the determined the fault signature vector $\varsigma$ has to be compared with the columns of $S$ according to

$$
\iota_{i}=\left\{\begin{array}{ll}
1 & \text { if } \varsigma=\bar{v}_{i} \\
0 & \text { otherwise }
\end{array} \text { for } i=1, \ldots, m_{f} .\right.
$$

The fault index vector $\iota$ has $m_{f}$ entries, where each entry $\iota_{i}$ indicates the presence (0) or absence (1) of the $i$-th fault.

\section{Determination of detection threshold}

To handle the requirements with respect to false alarms (FA) and missed detections (MD), the presented approach aims to choose decision thresholds which guarantee no false and partial firing. In case of an aircraft actuator, the whole nonlinear aircraft model has to be considered, to take all relevant parameter variations into account. To address the goal of no false firing the bound

$$
J_{t h, j}^{f}:=\sup \theta_{j}(t),
$$

can be defined, where the supremum is taken for all admissible operation points, all relevant aircraft maneuvers, all admissible variations of uncertain parameters, for all relevant disturbances as well as for all faults, which shall not generate a residual higher than the threshold. To avoid false firing, the threshold $J_{t h, j}$ used in the decision block must be chosen such that $J_{t h, j}>J_{t h, j}^{f}$. To address the goal of no partial firing, for each residual the bound

$$
J_{t h, j}^{d}:=\inf \theta_{j}(t),
$$

is defined, where the infinum involves all fault signals, which are supposed to generated a residual higher than the threshold. Note that these bounds implicitly limit the detectable fault amplitudes. To avoid partial firing, the threshold $J_{t h}$ used in the decision block must be chosen such that $J_{t h, j}<J_{t h, j}^{d}$. If $J_{t h, j}^{d}-J_{t h, j}^{f}>0$, a constant threshold $J_{t h, j}$ satisfying $J_{t h, j}^{d} \leq J_{t h, j}<J_{t h, j}^{f}$ can be chosen to guarantee no false as well as no partial firing. For the computation 
of $J_{t h, j}^{f}$ and $J_{t h, j}^{d}$, solving global worst-case optimization problems to find the worst-case parameter combinations is the most adequate choice [9].

\section{APPLICATION}

The developed FDD system including the residual filter is used to monitor an elevator actuator of a modern, augmented aircraft. Therefore, its functionality is validated simulating a nonlinear aircraft model including the nonlinear actuator dynamics (1). This allows a realistic validation of the FDD system, as the aerodynamic force acting on the actuator dynamics is updated at each time step using the simulation data of the aircraft model. For each sensor as well as for the active actuator a faulty situation is generated to illustrate the fault to residual transfer behavior in the nonlinear case. The sensors are corrupted with a sensor bias, while the actuator with a runaway. Note, due to the weak isolation characteristics, for fault isolation the mentioned faults have to occur one at a time.

The values of the evaluation function parameters (16) are selected at $\alpha_{j}=0.2, \beta_{j}=0.2$ and $\gamma_{j}=0.2$ for all $j=1, \ldots, 7$, providing a adequate filtering of the sensor noise. The thresholds are determined using worst case optimization and are set to $J_{t h}=\left\{J_{t h, 1}, \ldots, J_{t h, 7}\right\}=$ $\{0.5,0.5,0.5,0.5,0.5,0.5,5\}$. The last evaluated residual requires a higher threshold due to the determination of the derivative of the thirds sensor signal (see eq. (15)). On each of the two rod sensors a bias of $5 \mathrm{~mm}$, while on the sensor of the surface deflection a bias of $2.85 \mathrm{deg}$ is induced at $t_{f}=5 \mathrm{~s}$, which is approximately equivalent to a bias of $5 \mathrm{~mm}$ on the rod sensors. As actuator fault a runaway with a rate of around $20 \mathrm{deg} / \mathrm{s}$ is modeled.

Fig. 4 shows the behavior of the evaluated residuals during the different fault situations in the first four columns of the diagram, while in the last column the residual signals due to the input signal are depicted. The evaluated residuals $\theta_{j}$ are plotted in blue, the thresholds $J_{t h, j}$ in red and the elements $\varsigma_{j}$ of the fault signature vector $\varsigma$ in green. The residuals reflect the behavior coded in the DFSM 13). Fig. 5 finally shows the entries $\iota_{i}$ with $i=1, \ldots, 4$, of the fault index vector $\iota$, each one plotted with a different color. While during the four fault situations the correct fault is isolated, all signals of $\iota$ remain zero when the system is fault free, confirming the correct functionality of the proposed FDD system.

\section{CONCLUSION}

In this paper a systematic approach for the design of an FDD system for the detection and isolation of actuator and sensor faults in actuation systems of modern, augmented aircraft is proposed. The main steps of the methodology are: (1) the development of a suitable synthesis model of the underlying actuator, (2) the synthesis of residual generators for fault detection and isolation based on the desired fault signature matrix, and (3) the setting up of the fault evaluation and decision making including the determination of the detection thresholds. The designed residual generator has been adapted to cover a wide range of the flight envelope, still
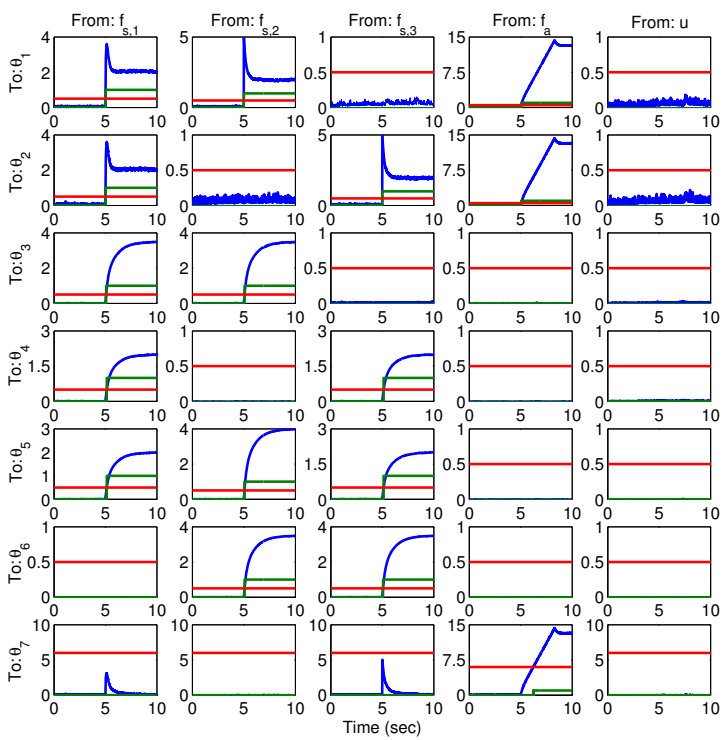

Fig. 4. Evaluated residuals due to the different faults

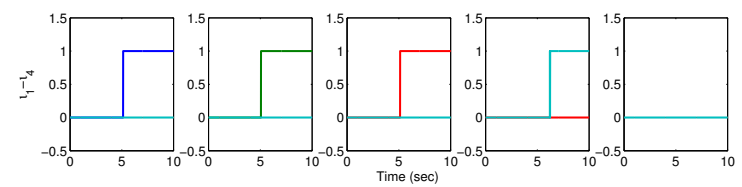

Fig. 5. Signals of the fault index vector $\iota$

showing a low complexity and therefore, low implementation costs. The validation of the FDD system in a nonlinear closed loop aircraft model indicates that the suggested FDD system cannot only reliably detect but also isolate the actuator and sensor faults. In this way, the proposed approach provides crucial information about the location of fault and thereby opens the gate to advanced fault tolerant control approaches on actuator level in the future.

\section{REFERENCES}

[1] E. A. Garcia, A. Zolghadri, P. Goupil, L. Lavigne and P. Simon. Nonlinear observer-based OFC detection for A380 aircraft In Proc. of IFAC Symp. SAFEPROCESS'2009 Barcelona, Spain, 2009.

[2] L. Van Eykeren, Q. Chu and J.A. Mulder. Actuator Fault Detection by Aerodynamic Model Identification. In Proc. of SAFEPROCESS'12 Mexico City, Mexico, 2012.

[3] A. Marcos. Application of H-infinity fault diagnosis to ADDSAFE benchmark: the control surface jamming case. In Proc. of AIAA Guidance, Navigation and Control Conference Portland, Oregon, 2011.

[4] B. Vanek, P. Seiler, J. Bokor and G. Balas. Robust Model Matching for Geometric Fault Detection Filters: A Commercial Aircraft Example. In Journal Automatic Control in Aerospace, 2011.

[5] L. Marton and D. Ossmann. Energetic Approach for Control Surface Disconnection Fault Detection in Hydraulic Aircraft Actuators. In Proc. of SAFEPROCESS'12 Mexico City, Mexico, 2012.

[6] A. Varga, S. Hecker and D. Ossmann. Diagnosis of actuator faults using LPV-gain scheduling techniques. In Proc. of AIAA Guidance, Navigation, and Control Conference Portland, Oregon, USA, 2011.

[7] A. Varga. On computing achievable fault signatures. In Proc. of IFAC Symp. SAFEPROCESS'2009 Barcelona, Spain, 2009.

[8] J. Gertler. Fault Detection and Diagnosis in Engineering Systems Marcel Dekker, New York, 1998.

[9] H. D. Joos. (1999). A methodology for multi-objective design assessment and flight control synthesis tuning. In Aerospace Science and Technology, 3, 161-176.

[10] A. Varga and D. Ossmann. LPV-model based identification approach of oscillatory failure cases. In Proc. of SAFEPROCESS'12 Mexico City, Mexico, 2012. 